\title{
miR-93 and PTEN: Key regulators of doxorubicin-resistance and EMT in breast cancer
}

\author{
SHIHUA CHU ${ }^{1 *}$, GENG LIU $^{2 *}$, PEIXUAN XIA $^{1}$, GUOQING CHEN $^{3}$, FENG SHI $^{1}$, \\ TAO $\mathrm{YI}^{4}$ and HONGYING ZHOU ${ }^{1}$ \\ ${ }^{1}$ Department of Human Anatomy, West China School of Preclinical and Forensic Medicine; \\ ${ }^{2}$ Division of Endocrinlogy and Metabolism, State Key Laboratory of Biotherapy, \\ West China Hospital and Collaborative Innovation Center of Biotherapy; \\ ${ }^{3}$ State Key Laboratory of Oral Diseases, West China Hospital of Stomatology; \\ ${ }^{4}$ Biotherapy Laboratory of Gynecological Oncology, Key Laboratory of Obstetric and Gynecologic \\ and Pediatric Diseases and Birth Defects of the Ministry of Education, West China Second Hospital, \\ Sichuan University, Chengdu, Sichuan 610041, P.R. China
}

Received March 5, 2017; Accepted July 10, 2017

DOI: 10.3892/or.2017.5859

\begin{abstract}
It is not well established whether miR-93 is involved in drug resistance and epithelial-mesenchymal transition (EMT) in breast cancer, and its underlying mechanism remains uncertain. In the present study, the expression differences of miR-93 between paired breast cancer tissues confirmed it is involved in the progression of breast cancer. Such a difference was also observed in doxorubicin-resistant and -sensitive cells. Overexpressed miR-93 in sensitive cells revealed increases in cellular proliferation and the expression levels of drug-resistant-related genes, and a decrease in sensitivity to doxorubicin. This demonstrated the relationship between miR-93 and breast cancer drug resistance. Simultaneously, EMT was confirmed in miR-93 overexpressing sensitive cells. This indicated the triadic relationship among miR-93, EMT and drug resistance in breast cancer. We applied the Dual-luciferase Reporter assay to expose the direct interaction between miR-93 and PTEN, which suggested that miR-93 contributes to inducing
\end{abstract}

Correspondence to: Professor Hongying Zhou, Department of Human Anatomy, West China School of Preclinical and Forensic Medicine, Sichuan University, 17, The 3rd section, Renmin Road, Chengdu, Sichuan, P.R. China

E-mail: eaglezhyxzy@163.com

Professor Tao Yi, Biotherapy Laboratory of Gynecological Oncology, Key Laboratory of Obstetric and Gynecologic and Pediatric Diseases and Birth Defects of the Ministry of Education, West China Second Hospital, Sichuan University, 17, The 3rd section, Renmin Road, Chengdu, Sichuan 610041, P.R. China E-mail: yitao-yt@hotmail.com

*Contributed equally

Key words: miR-93, EMT, PTEN, doxorubicin-resistance
EMT and drug resistance of breast cancer cells by targeting PTEN.

\section{Introduction}

Breast cancer is the leading cause of cancer-related mortality among women (1). This high incidence of mortality is, to a large extent, due to drug-resistance, which is the major obstacle to successful clinical treatment (2).

Numerous studies have demonstrated that microRNAs (miRNAs) are involved in the process of epithelial-mesenchymal transition (EMT) in various types of cancer (3-6), and various studies have revealed that the EMT may be associated with the drug resistance of cancer cells (7-10). Furthermore, by comparing the miRNA expression profiles between breast cancer cell lines in our previous study, we determined that the expression of miR-93 was increased markedly in drug-resistant MCF-7/AdrVp cells compared with the parental MCF-7 cell line (11).

Numerous studies on breast cancer have demonstrated that PTEN is involved in EMT $(5,12)$ and in the drug resistance of cancer cells $(6,13)$. Although a few studies have shown that miR-93 may functionally interact with PTEN, this has only been reported in cardiomyocyte apoptosis, osteosarcoma, ovarian cancer, glioma, hepatocellular carcinoma and prostate cancer (14-20). Therefore, it remains to be determined whether miR-93 is also functionally associated with PTEN in breast cancer, and whether such an association contributes to the induction of EMT and drug resistance in breast cancer cells. The present study aimed to clarify the role of miR-93 in drug resistance and EMT in breast cancer, and to investigate its target gene.

\section{Materials and methods}

Tissue samples. Sixteen pairs of tissue samples of ductal breast cancer were obtained from the West China Hospital of 
Sichuan University. All the patients underwent surgical resection to obtain the breast cancerous and corresponding adjacent non-tumorous tissues, without receiving chemotherapy or radiotherapy beforehand. All tissues were preserved in liquid nitrogen. The present study was approved by the local ethical standards of the Institutional Review Board of Sichuan University. Informed consent was obtained from all individual participants included in the present study.

Ethical approval. All procedures performed in the present study involving human participants were in accordance with the ethical standards of the Institutional and/or National Research Committee of Sichuan University and with the 1964 Helsinki declaration and its later amendments or comparable ethical standards.

Cell lines and transfections. The MCF-7 cell line was purchased from the Shanghai Institutes for Biological Sciences (Shanghai, China) and cultured according to a protocol from the ATCC.

The doxorubicin-resistant MCF-7/ADR cell line (MCF-7/ADR) was induced by continuously culturing MCF-7 cells in medium containing progressive concentrations of doxorubicin (Sigma-Aldrich, St. Louis, MO, USA). MCF-7/ADR cells were cultured in medium with $2 \mu \mathrm{g} / \mathrm{ml}$ doxorubicin, and subsequently transferred into a drug-free medium for at least 2-3 weeks before use in the assays.

Using Lipofectamine 3000 (Invitrogen, Carlsbad, CA, USA) according to the manufacturer's instructions, MCF-7 cells were transfected with $50 \mathrm{nM}$ hsa-miR-93-5p mimics or hsa-miR-93-5p negative control mimics (RiboBio Co., Ltd., Guangzhou, China) in order to produce an MCF-7-miR-93 mimics group (MCF-7-miR-93 mimics) and a negative control group (MCF-7-miR-93 mimics NC), respectively. In addition, MCF-7/ADR cells were transfected with $100 \mathrm{nM}$ hsa-miR-93-5p inhibitor (RiboBio Co., Ltd.) to produce an MCF-7/ADR-miR-93 inhibitor group, or an hsa-miR-93-5p inhibitor negative control (RiboBio Co., Ltd.) to produce an MCF-7/ADR-miR-93 inhibitor NC group, following the same procedure as aforementioned.

$R N A$ isolation and $q R T-P C R$. Total miRNAs were extracted from cells and tissue samples using an miRcute miRNA Isolation kit (Tiangen Biotech Co., Ltd., Beijing, China). The expression of miR-93 was analyzed using the Bulge-Loop ${ }^{\mathrm{TM}}$ miRNA qRT-PCR Starter kit (RiboBio Co., Ltd.). The primers for miR-93 and endogenous control U6 were purchased from RiboBio Co., Ltd. (ssD809230675, ssD809231367, ssD809261711, ssDD0904071006, ssDD0904071007 and ssDD0904071008).

TRIzol reagent (Takara, Dalian, China) was used for total RNA extraction. The expression of different genes was analyzed using a SYBR-Green qRT-PCR kit (Takara). The primer sequences for qRT-PCR were as follows: E-cadherin, 5'-tgcccagaaaatgaaaaagg-3', 5'-gtgtatgtggcaatgcgttc-3' (product size, 200 bp); vimentin, 5'-gagaactttgccgttgaagc-3, 5'-tccagcagcttcctgtaggt-3' (product size, $170 \mathrm{bp}$ ); N-cadherin, 5'-gac aatgcccctcaagtgtt-3', 5'-ccattaagccgagtgatggt-3' (product size, $179 \mathrm{bp}$ ); fibronectin, 5'-accaacctacggatgactcg-3', 5'-gctcatcatc tggccatttt-3' (product size, $230 \mathrm{bp}$ ); Snail, 5'-ggttcttctgcgctactgct-3', 5'-tagggctgctggaaggtaaa-3' (product size, $157 \mathrm{bp}$ );
Twist, 5'-ggagtccgcagtcttacgag-3', 5'-tggaggacctggtagaggaa-3' (product size, 199 bp); MRP, 5'-aggtggacctgtttcgtgac-3', 5'-cctg tgatccaccagaaggt-3' (product size, $181 \mathrm{bp}$ ); BCRP, 5'-caccttattg gcctcaggaa-3', 5'-cctgcttggaaggctctatg-3' (product size, 206 bp); MDR, 5'-gctcctgactatgccaaagc-3', 5'-tcttcacctccaggctcagt-3' (product size, 202 bp); PTEN, 5'-ttacagttgggecctgtacc-3', 5'-atttgatgetgccggtaaac-3' (product size, $153 \mathrm{bp}$ ); GAPDH, 5'-ct ttggtatcgtggaaggactc-3', 5'-gtagaggcagggatgatgttct-3' (product size, $132 \mathrm{bp})$.

Flow cytometry. Cell apoptosis was detected using an Annexin V-FITC apoptosis detection kit (KeyGen Biotech Co., Ltd., Nanjing, China), according to the manufacturer's instructions. The data were analyzed using FlowJo 9.1 software.

CCK-8 assay. Cellular proliferation and growth inhibition were assessed using Cell Counting Kit-8 (CCK-8) assay (Dojindo Laboratories, Kumamoto, Japan) according to the manufacturer's protocol. For the detection of the proliferation of cells in the MCF-7-miR-93 mimic group, the cells were assessed at 0, 24, 48 and $72 \mathrm{~h}$. In addition, to determine the effect of miR-93 on drug resistance, the cell survival ratio was assessed after cells in the MCF-7-miR-93 mimic group were cultured with $0.08,0.4,1$ and $5 \mu \mathrm{g} / \mathrm{ml}$ doxorubicin (Sigma-Aldrich) for $24 \mathrm{~h}$.

Dual-luciferase reporter assay. A bioinformatics analysis using the 'miRanda' database was performed to analyze the possibility of miR-93 binding to PTEN. Subsequently, MCF-7 cells were cotransfected with $2.5 \mu \mathrm{g}$ pGL3 luciferase reporter plasmid (GeneCopoeia) containing either a wild-type (5'-GGA UUAAUAAAGAUGGCACUUUC-3') or mutated (5'-GGAUU AAUAAAGAUGGCTCUAUC-3') form of the PTEN 3'-UTR, and $50 \mathrm{nM}$ hsa-miR-93-5p or miR-93 mimics NC in 6-well plates using Lipofectamine 3000. Luciferase activity was assessed consecutively at $24 \mathrm{~h}$ post-transfection using a DualLuciferase Assay (GeneCopoeia), and normalized to the blank vector control group.

Statistical analysis. All the experiments were repeated three times independently. Data were calculated as the mean \pm SD. The paired t-test was applied for statistical analysis using SPSS software (version 20.0) (SPSS, Inc., Chicago, IL, USA).

\section{Results}

Expression of miR-93 in breast cancer in vivo and in vitro. qRT-PCR was performed to identify the patterns of miR-93 expression in breast cancer tissues and a doxorubicin-resistant breast cancer cell line (MCF-7/ADR) compared with paired normal breast tissue samples and a doxorubicin-sensitive parental breast cancer cell line (MCF-7). The results revealed that $62.5 \%$ of cancer tissue samples (10 out of 16 cases) exhibited a markedly higher expression level of miR-93 compared with their corresponding paired adjacent normal tissue (Fig. 1A; $p<0.05$ ). Furthermore, the miR-93 expression level in the MCF-7/ADR cells was significantly higher than that in MCF-7 cells (Fig. 1B; $\mathrm{p}<0.01$ ).

Proliferation and doxorubicin-resistance of MCF-7 cells transfected with miR-93 mimics. A CCK-8 assay was performed 

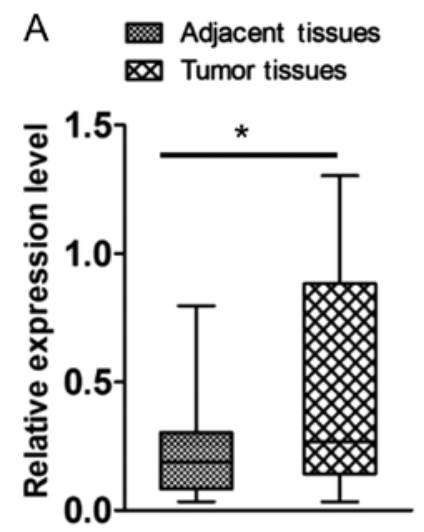

B $\quad$ MCF-7

区 MCF-7/ADR

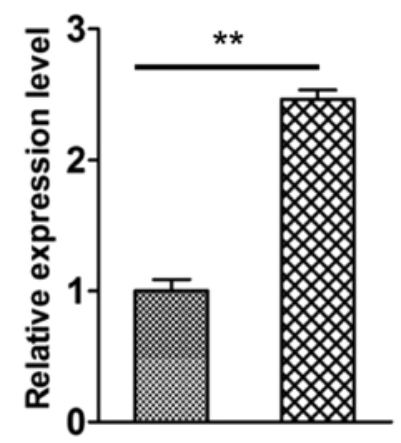

Figure 1. Expressions of miR-93 in breast cancer in vivo and vitro. (A) The expression level of miR-93 was higher in breast cancer tissues compared to the adjacent normal tissues ( $\mathrm{n}=16$ ). (B) MCF-7/ADR cells showed a significantly higher expression level of miR-93 than the MCF-7 cells; ${ }^{*} \mathrm{p}<0.05,{ }^{* *} \mathrm{p}<0.01$.

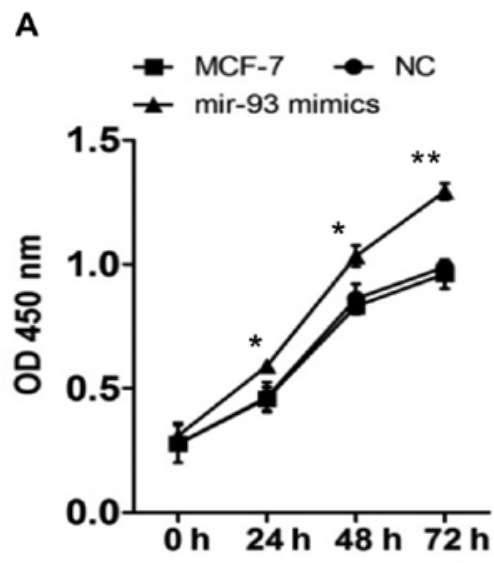

B

MCF-7 四 NC miR-93 mimics

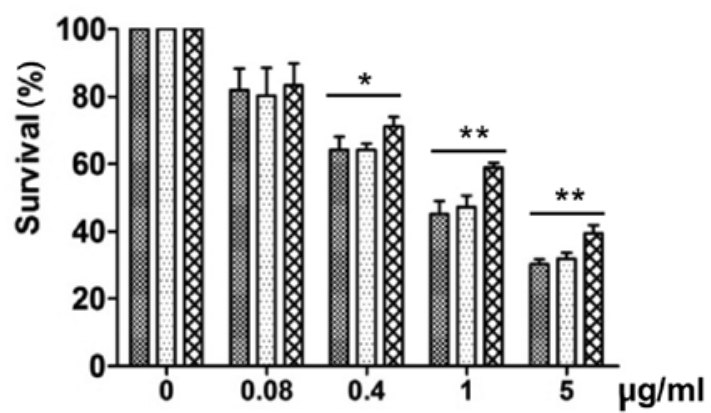

C

MCF-7 四 NC miR-93 mimics

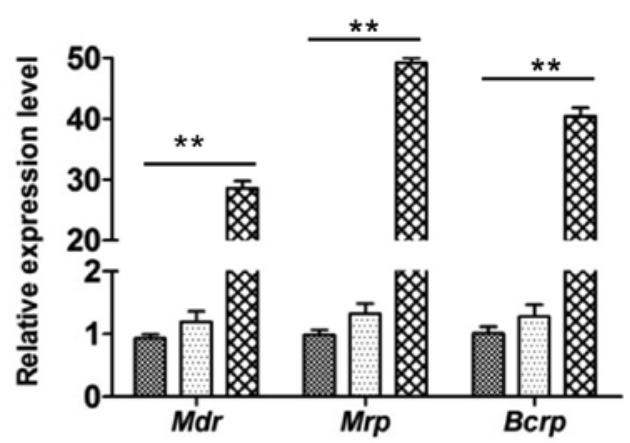

Figure 2. Effects of miR-93 on cell proliferation, cell survival and drug resistance. (A) Cell proliferation was determined by CCK- 8 assay. At $0,24,48$ and $72 \mathrm{~h}$, the OD value in each group of cells was assessed by the absorbance at $450 \mathrm{~nm}$. (B) Overexpression of miR-93 in MCF-7 cells increased the survival ratio. Cell viability was determined by CCK-8 assay. The MCF-7-miR-93 mimic cells and its control cells were treated with $0.08,0.4,1$ and $5 \mu \mathrm{g} / \mathrm{ml}$ doxorubicin for $24 \mathrm{~h}$. The percentage of viable cells was normalized to that of the untreated controls. (C) Overexpression of miR-93 increased the expression of multi-drug resistant marker genes (MDR, MRP and BCRP) in MCF-7 cells; ${ }^{*} \mathrm{p}<0.05,{ }^{* *} \mathrm{p}<0.01$.

to evaluate the proliferation and sensitivity to doxorubicin of cells in the MCF-7-miR-93 mimics group. The results revealed that the overexpression of miR-93 markedly upregulated the proliferation rate of MCF-7 cells (Fig. 2A; p<0.01), and also significantly increased the survival ratio of cells treated with doxorubicin for $24 \mathrm{~h}$ (Fig. 2B; p<0.05).

qRT-PCR was used to examine the expression levels of the multi-drug resistant-related genes MDR, MRP and BCRP in
MCF-7 and MCF-7-miR-93 mimic cells. The results demonstrated that the expression levels of each of these genes were significantly higher in the MCF-7-miR-93 mimic cells than in the MCF-7 cells (Fig. 2C; p<0.01). Furthermore, flow cytometric analysis revealed that the rate of doxorubicin-induced apoptosis was lower in the MCF-7-miR-93 mimic cells than in untransfected MCF-7 cells (Fig. 3A, B and E; p<0.01). By contrast, in the MCF-7/ADR-miR-93 inhibitor cells, the rate 

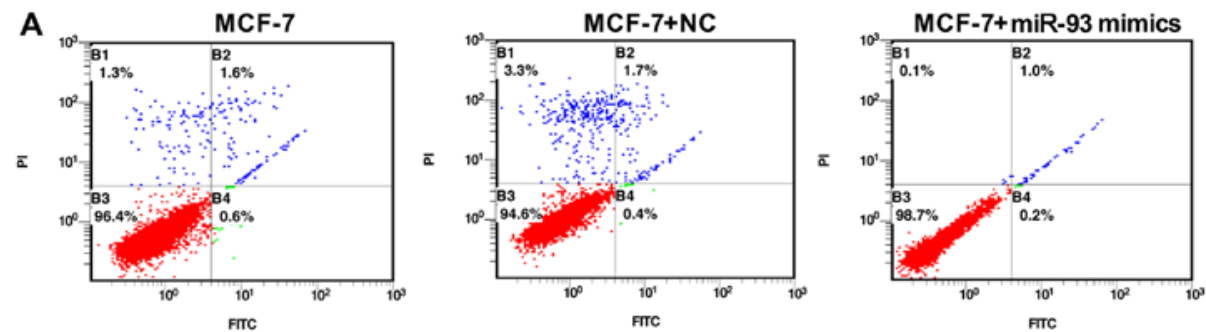

B
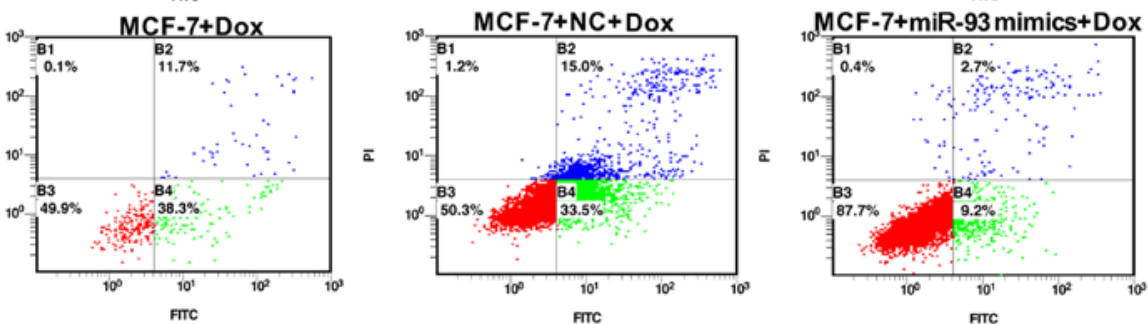

C

FTC
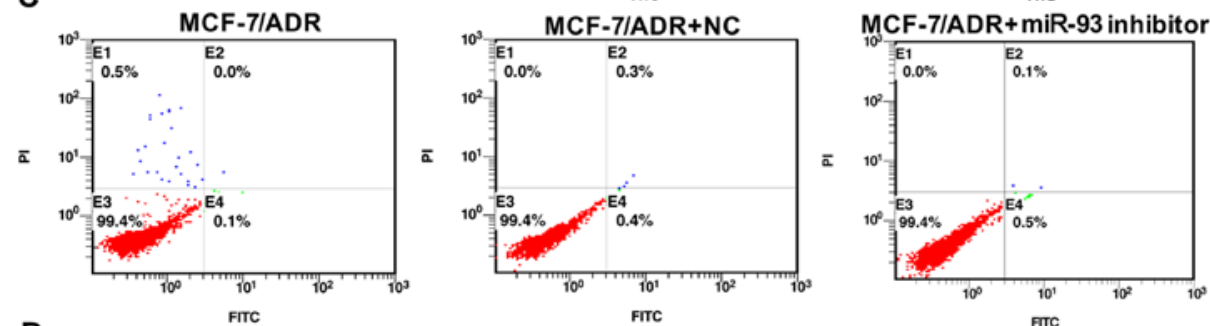

D
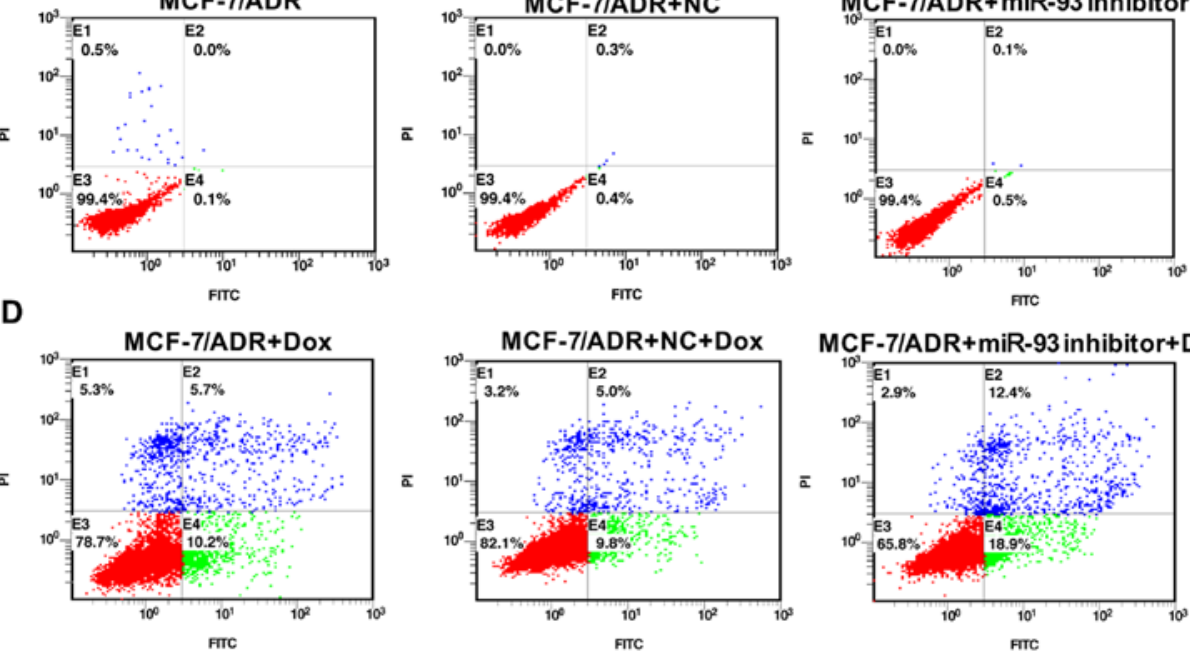

MCF-7/ADR+miR-93 inhibitor+Dox

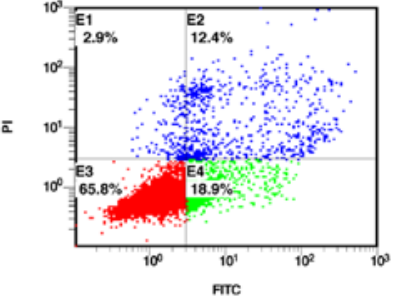

E NC+Dox $\otimes$ miR-93 mimics+Dox

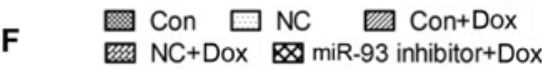

F Con $\square \mathrm{NC}$ 罚 Con+Dox

Con+Dox
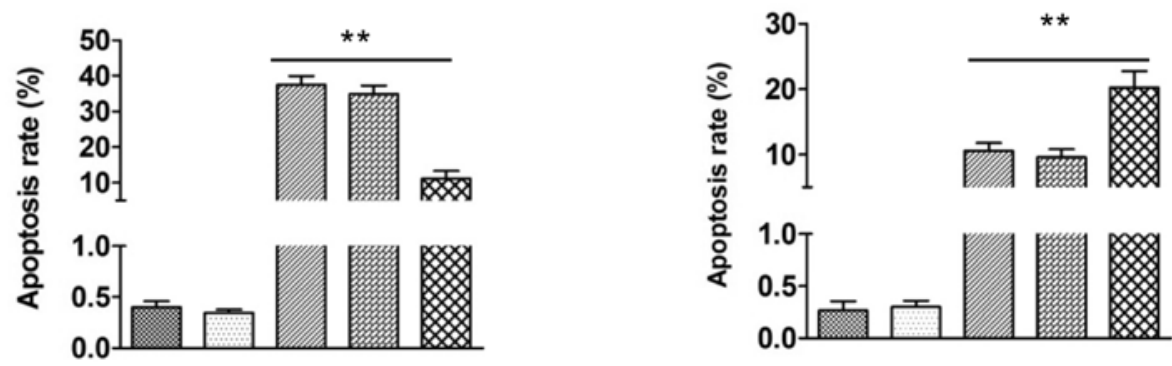

Figure 3. Flow cytometric analysis of the miR-93 effect on doxorubicin-induced apoptosis in breast cancer cells. Cell apoptosis was induced by $1 \mu \mathrm{g} / \mathrm{ml}$ doxorubicin in MCF-7 cells, and $10 \mu \mathrm{g} / \mathrm{ml}$ doxorubicin in MCF-7/ADR cells. (A and C) MCF-7 and MCF-7/ADR cell apoptotic rate was barely without the doxorubicin treatment. (B and E) The proportion of apoptotic cells was lower in the MCF-7-miR-93 mimic cells than that in the control; (D and F) while it was higher in the MCF-7/ADR-miR-93 inhibitor cells than that in the control; ${ }^{* *} \mathrm{p}<0.01$.

of doxorubicin-induced apoptosis was significantly increased compared with that in the untransfected MCF-7/ADR-miR-93 cells (Fig. 3C, D, and F; p<0.01).

EMT in MCF-7 cells overexpressing miR-93. To explore the possible relationship between miR-93 expression and cancer cell EMT, the changes in morphological features and the mRNA levels of EMT-related genes were examined after the transfection of MCF-7 cells with miR-93 mimics or NC. The morphological features of the cells are shown in Fig. 4A. MCF-7-miR-93 mimic-transfected cells displayed a cobbleston-like appearance and tight cell-cell junctions. By contrast, the MCF-7-miR-93 mimic NC and untransfected MCF-7 cells appeared to have spindle-cell morphology. Meanwhile, the qRT-PCR results revealed that the expression level of the epithelial marker E-cadherin was markedly decreased, while the levels of the mesenchymal markers N-cadherin, vimentin, Twist, Snail and fibronectin were markedly increased after transfection with the miR-93 mimics (Fig. 4B; p $<0.01$ ).

Dual-luciferase reporter assay and qRT-PCR analysis of $P T E N$. To detect the possible association between miR-93 
A
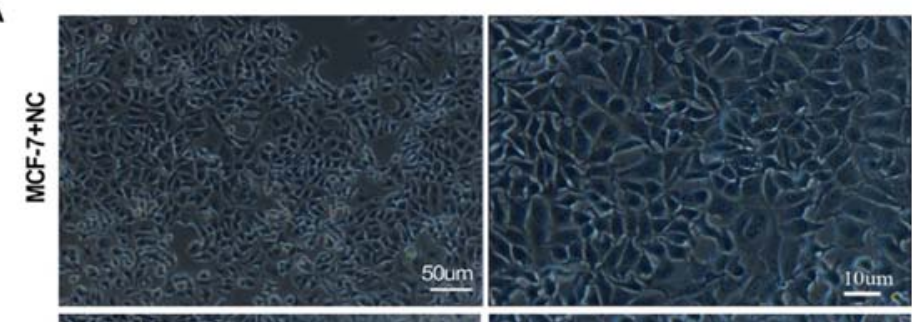

苍
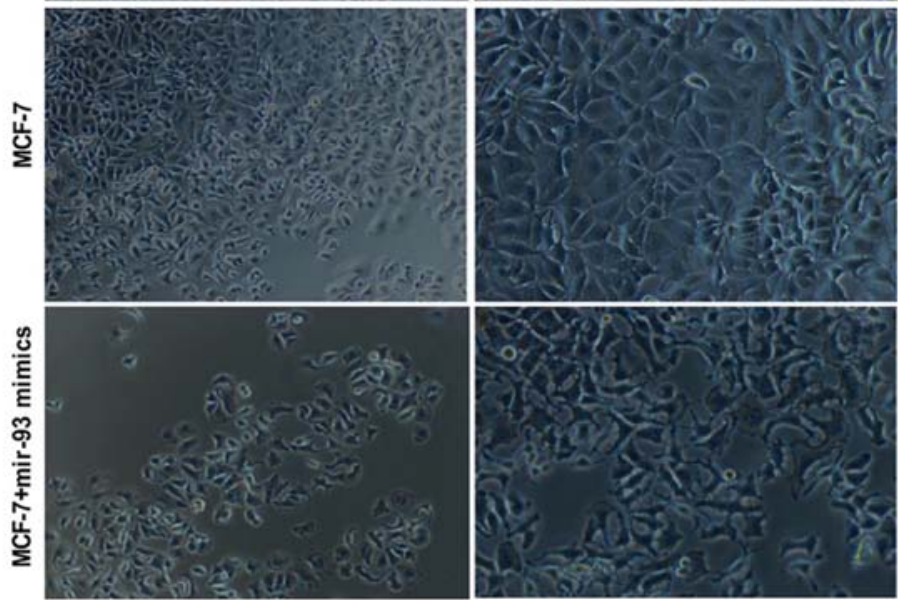

B

MCF-7 NC $\square$ miR-93 mimics

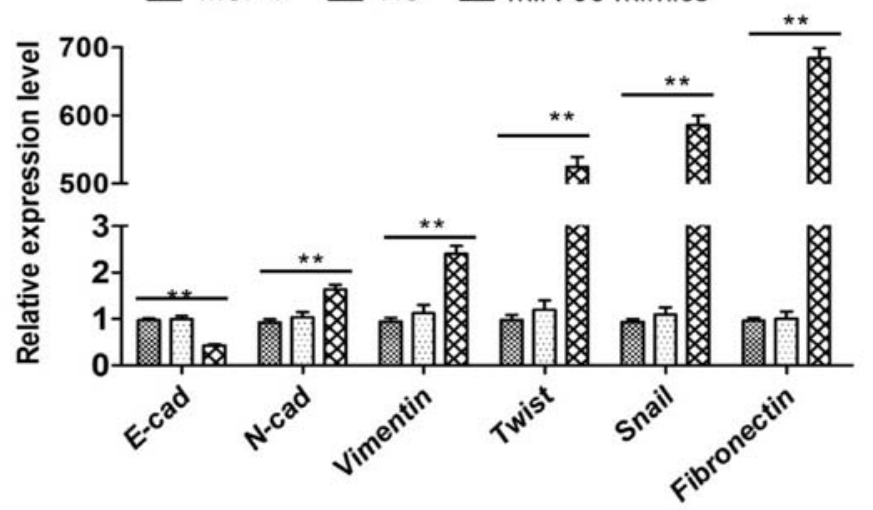

Figure 4. Overexpression of miR-93 contributes to EMT progression in MCF-7 cells. (A) The morphological characteristics of the miR-93-overexpressed MCF-7 cells and its negative control. MCF-7-miR-93 mimic cells displayed a cobblestone-like appearance and tight cell-cell junction. In contrast, the MCF-7-miR-93 NC cells appeared to have spindle-cell morphology. (B) qRT-PCR analysis of EMT-related marker genes (E-cad, N-cad, vimentin, Twist, Snail and fibronectin) mRNA expression level; ${ }^{*} \mathrm{p}<0.05,{ }^{* *} \mathrm{p}<0.01$.

A

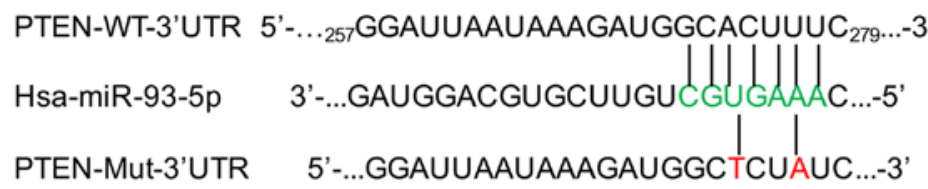

B

$\square$ Vector $\square$ PTEN-WT-3'UTR XPTEN-MUt-3'UTR

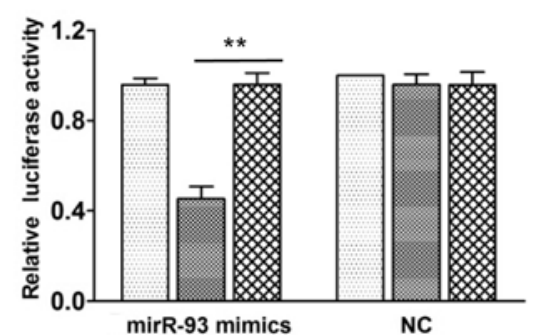

C

$\square$ MCF-7 $\square$ NC

$\otimes$ miR-93 mimics

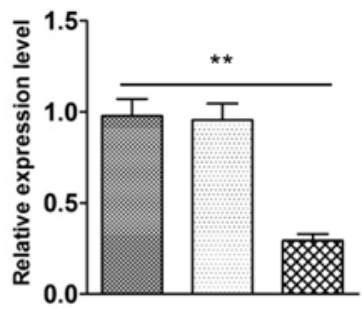

Figure 5. miR-93 directly targets PTEN. (A) The potential binding site of miR-93 in the 3'-UTR of PTEN. (B) The luciferase activity in the PTEN-WT-3'-UTR was significantly lower than the PTEN-Mut-3'-UTR control when cotransfected with miR-93 mimics. (C) Overexpression of miR-93 decreased the PTEN expression as determined by qRT-PCR analysis; ${ }^{*} \mathrm{p}<0.05,{ }^{* *} \mathrm{p}<0.01$. 
and PTEN, a 'miRanda' bioinformatics analysis was initially performed. The miRNA target prediction program indicated PTEN as one of the possible target genes of miR-93. In particular, the 3'-untranslated region (UTR) of PTEN mRNA contains a binding site for miR-93 (Fig. 5A). To confirm this, a dual-luciferase reporter assay was carried out. The results revealed that,compared with the control group (MCF-7-miR-93 mimic NC), transfection with the miR-93 mimics decreased the luciferase activity of the reporter construct containing the wild-type PTEN 3'-UTR (Fig. 5B; p<0.01), whereas the miR-93 mimics induced no significant change in the activity of the reporter construct containing the mutated PTEN 3'-UTR. This indicated that miR-93 can bind directly to the PTEN 3'-UTR. Furthermore, the qRT-PCR results confirmed that the miR-93 mimics could downregulate the expression level of PTEN in MCF-7 cells compared with the MCF-7-miR-93 mimics NC group (Fig. 5C; $\mathrm{p}<0.01$ ).

\section{Discussion}

It has been widely reported that miRNAs are involved in numerous molecular events in various types of tumors (21-23), including the acquisition of drug resistance, which is one of the most prominent clinical challenges at present. In the present study, miR-93 was selected for investigation as its function remains uncertain in many contexts, particularly in breast cancer. We aimed to clarify the role of miR-93 in the acquisition of drug resistance of breast cancer cells.

miR-93, along with miR-106b and miR-25, is a member of the miR-106b-25 cluster, which is located in its host gene, MCM7 (24). All members of the miR-106b-25 cluster, in addition to MCM7, have been reported to be involved in tumorigenesis $(25-27)$ and drug resistance $(28,29)$ in multiple tumors. Furthermore, our previous study on miRNA profiles revealed that miR-93 was the most upregulated miRNA of this cluster in the doxorubicin-resistant MCF-7 cells compared with the parental MCF-7 cells, which indicated that miR-93 may be the major contributor to the drug resistance of breast cancer cells in this cluster. Simultaneously, in our preliminary experiments, we also tested the differential expression trends of MCM7 in MCF-7/ADR and MCF-7 cells, which revealed a less marked difference than that of miR-93. Therefore, miR-93, rather than the whole cluster and its host gene, may play a critical role in the acquisition of drug resistance in breast cancer. To date, very few studies have focused on the role of miR-93 in breast cancer (30-36). Furthermore, these studies only reported its association with altered expression patterns and proliferation, and none have focused on its relationship with drug resistance and association with PTEN.

The results of the present study revealed a higher expression level of miR-93 in primary ductal breast cancer tissues than in corresponding tumor-adjacent normal tissues, and an increased proliferation rate in miR-93-overexpressing MCF-7 cells, which confirmed that miR-93 may be involved in the progression of breast cancer. Furthermore, a higher expression level of miR-93 was observed in MCF-7/ADR cells than in MCF-7 cells, and the survival ratio of miR-93-overexpressing MCF-7 cells following exposure to doxorubicin was increased markedly compared with that of the miR-93 mimics NC group. Furthermore, the expression levels of multi-drug resistance-related genes were significantly upregulated in the miR-93-overexpressing MCF-7 cells compared with NC-transfected cells, concomitant with the increase in doxorubicin resistance. In contrast, the miR-93 inhibitor treatment led to a reversal of doxorubicin resistance in the MCF-7/ADR cells. Thus, we suggest that miR-93 may contribute to the doxorubicin resistance of breast cancer cells.

EMT has been observed in the majority of tumors $(37,38)$, and emerging evidence also indicates that it may be involved in drug resistance in certain types of cancer cells $(7,39)$, including breast cancer $(40,41)$. In the present study, the observed morphological changes and the upregulation of EMT-related genes in miR-93-overexpressing MCF-7 cells indicated that miR-93 participates in EMT in breast cancer cells. Moreover, the significant upregulation of drug resistance-related genes in the miR-93-overexpressing MCF-7 cells was observed concomitantly with EMT, which infers that EMT may be involved in the miR-93-induced drug resistance of breast cancer cells.

Following bioinformatics analysis and the retrieval of related literature (18), we speculated that PTEN may be a direct target gene of miR-93, and the results of a dual-luciferase reporter assay and the downregulation of PTEN in miR-93-overexpressing MCF-7 cells confirmed this direct interaction in breast cancer cells. The PI3K/Akt signaling pathway is considered to be one of the mechanisms underlying EMT in cancer cells, and PTEN has been shown to be a regulatory factor upstream of this pathway (42). Combined with our results, we hypothesize that miR-93 affects EMT through its interaction with PTEN, subsequently inducing drug resistance in breast cancer cells.

In conclusion, miR-93 may play an important role in EMT and drug resistance of breast cancer cells by targeting PTEN. The present study provides novel insights into the biological function of miR-93 in breast cancer drug resistance. miR-93 may be considered a potential biomarker of prognosis, and a promising therapeutic target for the reversal of drug resistance in patients with breast cancer.

\section{References}

1. Siegel RL, Miller KD and Jemal A: Cancer statistics, 2015. CA Cancer J Clin 65: 5-29, 2015.

2. Holohan C, Van Schaeybroeck S, Longley DB and Johnston PG: Cancer drug resistance: An evolving paradigm. Nat Rev Cancer 13: 714-726, 2013.

3. Lamouille S, Subramanyam D, Blelloch R and Derynck R: Regulation of epithelial-mesenchymal and mesenchymalepithelial transitions by microRNAs. Curr Opin Cell Biol 25: 200-207, 2013.

4. Zaravinos A: The Regulatory Role of MicroRNAs in EMT and Cancer. J Oncol 2015: 865816, 2015.

5. Li C, Song L, Zhang Z, Bai XX, Cui MF and Ma LJ: MicroRNA-21 promotes TGF- $\beta 1$-induced epithelial-mesenchymal transition in gastric cancer through up-regulating PTEN expression. Oncotarget 7: 66989-67003, 2016.

6. Li H, Zhang P, Sun X, Sun Y, Shi C, Liu H and Liu X: MicroRNA-181a regulates epithelial-mesenchymal transition by targeting PTEN in drug-resistant lung adenocarcinoma cells. Int J Oncol 47: 1379-1392, 2015.

7. Brozovic A: The relationship between platinum drug resistance and epithelial-mesenchymal transition. Arch Toxicol 91: 605-619, 2017.

8. Huang J, Li H and Ren G: Epithelial-mesenchymal transition and drug resistance in breast cancer (Review). Int J Oncol 47: 840-848, 2015 
9. Du B and Shim JS: Targeting epithelial-mesenchymal transition (EMT) to overcome drug resistance in cancer. Molecules 21: pii: E965, 2016.

10. Bugide S, Gonugunta VK, Penugurti V, Malisetty VL, Vadlamudi RK and Manavathi B: HPIP promotes epithelialmesenchymal transition and cisplatin resistance in ovarian cancer cells through PI3K/AKT pathway activation. Cell Oncol 40: 133-144, 2016.

11. Chen GQ, Zhao ZW, Zhou HY, Liu YJ and Yang HJ: Systematic analysis of microRNA involved in resistance of the MCF-7 human breast cancer cell to doxorubicin. Med Oncol 27: 406-415, 2010.

12. Miao Y, Zheng W, Li N, Su Z, Zhao L, Zhou H and Jia L: MicroRNA-130b targets PTEN to mediate drug resistance and proliferation of breast cancer cells via the PI3K/Akt signaling pathway. Sci Rep 7: 41942, 2017.

13. Xia H, Ooi LL and Hui KM: MicroRNA-216a/217-induced epithelial-mesenchymal transition targets PTEN and SMAD7 to promote drug resistance and recurrence of liver cancer. Hepatology 58: 629-641, 2013.

14. Chen Q, Qin R, Fang Y and Li H: Berberine sensitizes human ovarian cancer cells to cisplatin through miR-93/PTEN/Akt signaling pathway. Cell Physiol Biochem 36: 956-965, 2015.

15. Ke ZP, Xu P, Shi Y and Gao AM: MicroRNA-93 inhibits ischemia-reperfusion induced cardiomyocyte apoptosis by targeting PTEN. Oncotarget 7: 28796-28805, 2016.

16. Fu X, Tian J, Zhang L, Chen Y and Hao Q: Involvement of microRNA-93, a new regulator of PTEN/Akt signaling pathway, in regulation of chemotherapeutic drug cisplatin chemosensitivity in ovarian cancer cells. FEBS Lett 586: 1279-1286, 2012

17. Jiang L, Wang C, Lei F, Zhang L, Zhang X, Liu A, Wu G, Zhu J and Song L: miR-93 promotes cell proliferation in gliomas through activation of PI3K/Akt signaling pathway. Oncotarget 6 : 8286-8299, 2015.

18. Ohta K, Hoshino H, Wang J, Ono S, Iida Y, Hata K, Huang SK Colquhoun S and Hoon DS: MicroRNA-93 activates c-Met/PI3K/ Akt pathway activity in hepatocellular carcinoma by directly inhibiting PTEN and CDKN1A. Oncotarget 6: 3211-3224, 2015.

19. McCann MJ, Rowland IR and Roy NC: The anti-proliferative effects of enterolactone in prostate cancer cells: Evidence for the role of DNA licencing genes, mi-R106b cluster expression, and PTEN dosage. Nutrients 6: 4839-4855, 2014.

20. Kawano M, Tanaka K, Itonaga I, Ikeda S, Iwasaki $T$ and Tsumura H: microRNA-93 promotes cell proliferation via targeting of PTEN in Osteosarcoma cells. J Exp Clin Cancer Res 34: 76, 2015.

21. Shen J, Stass SA and Jiang F: MicroRNAs as potential biomarkers in human solid tumors. Cancer Lett 329: 125-136, 2013.

22. Bertoli G, Cava C and Castiglioni I: MicroRNAs: New biomarkers for diagnosis, prognosis, therapy prediction and therapeutic tools for breast cancer. Theranostics 5: 1122-1143, 2015

23. An X, Sarmiento C, Tan T and Zhu H: Regulation of multidrug resistance by microRNAs in anti-cancer therapy. Acta Pharm Sin B 7: 38-51,2017.

24. Poliseno L, Salmena L, Riccardi L, Fornari A, Song MS, Hobbs RM, Sportoletti P, Varmeh S, Egia A, Fedele G, et al: Identification of the miR-106b 25 microRNA cluster as a protooncogenic PTEN-targeting intron that cooperates with its host gene $M C M 7$ in transformation. Sci Signal 3: ra29, 2010.

25. Petrocca F, Visone R, Onelli MR, Shah MH, Nicoloso MS, de Martino I, Iliopoulos D, Pilozzi E, Liu CG, Negrini M, et al: E2F1-regulated microRNAs impair TGFbeta-dependent cell-cycle arrest and apoptosis in gastric cancer. Cancer Cell 13: 272-286, 2008

26. Gong C, Qu S, Liu B, Pan S, Jiao Y, Nie Y, Su F, Liu Q and Song E: MiR-106b expression determines the proliferation paradox of TGF- $\beta$ in breast cancer cells. Oncogene 34: 84-93, 2015.
27. Chen S, Chen X, Xiu YL, Sun KX and Zhao Y: Inhibition of ovarian epithelial carcinoma tumorigenesis and progression by microRNA 106b mediated through the RhoC pathway. PLoS One 10: e0125714, 2015

28. Zhou Y, Hu Y, Yang M, Jat P, Li K, Lombardo Y, Xiong D, Coombes RC, Raguz S and Yagüe E: The miR-106b 25 cluster promotes bypass of doxorubicin-induced senescence and increase in motility and invasion by targeting the E-cadherin transcriptional activator EP300. Cell Death Differ 21: 462-474, 2014.

29. Hu Y, Li K, Asaduzzaman M, Cuella R, Shi H, Raguz S, Coombes RC, Zhou Y and Yagüe E: MiR-106b 25 cluster regulates multidrug resistance in an $\mathrm{ABC}$ transporter-independent manner via downregulation of EP300. Oncol Rep 35: $1170-1178,2016$

30. Kolacinska A, Morawiec J,Pawlowska Z, Szemraj J, Szymanska B, Malachowska B, Morawiec Z, Morawiec-Sztandera A, Pakula L, Kubiak R, et al: Association of microRNA-93, 190, 200b and receptor status in core biopsies from stage III breast cancer patients. DNA Cell Biol 33: 624-629, 2014.

31. McDermott AM, Miller N, Wall D, Martyn LM, Ball G, Sweeney KJ and Kerin MJ: Identification and validation of oncologic miRNA biomarkers for luminal A-like breast cancer. PLoS One 9: e87032, 2014

32. Hu J, Xu J, Wu Y, Chen Q, Zheng W, Lu X, Zhou C and Jiao D: Identification of microRNA-93 as a functional dysregulated miRNA in triple-negative breast cancer. Tumour Biol 36: 251-258, 2015.

33. Liu S, Patel SH, Ginestier C, Ibarra I, Martin-Trevino R, Bai S, McDermott SP, Shang L, Ke J, Ou SJ, et al: MicroRNA93 regulates proliferation and differentiation of normal and malignant breast stem cells. PLoS Genet 8: e1002751, 2012.

34. Singh B, Ronghe AM, Chatterjee A, Bhat NK and Bhat HK: MicroRNA-93 regulates NRF2 expression and is associated with breast carcinogenesis. Carcinogenesis 34: 1165-1172, 2013.

35. Smith AL, Iwanaga R, Drasin DJ, Micalizzi DS, Vartuli RL, Tan AC and Ford HL: The miR-106b-25 cluster targets Smad7, activates TGF- $\beta$ signaling, and induces EMT and tumor initiating cell characteristics downstream of Six1 in human breast cancer. Oncogene 31: 5162-5171, 2012.

36. Fang L, Du WW, Yang W, Rutnam ZJ, Peng C, Li H, O'Malley YQ, Askeland RW, Sugg S, Liu M, et al: MiR-93 enhances angiogenesis and metastasis by targeting LATS2. Cell Cycle 11: 4352-4365, 2012

37. Yeung KT and Yang J: Epithelial-mesenchymal transition in tumor metastasis. Mol Oncol 11: 28-39, 2017.

38. Xiang J, Fu X, Ran W and Wang Z: Grhl2 reduces invasion and migration through inhibition of TGF $\beta$-induced EMT in gastric cancer. Oncogenesis 6: e284, 2017.

39. Dia VP and Pangloli P: Epithelial-to-mesenchymal transition in paclitaxel-resistant ovarian cancer cells is downregulated by luteolin. J Cell Physiol 232: 391-401, 2017.

40. Preca BT, Bajdak K, Mock K, Lehmann W, Sundararajan V, Bronsert P, Matzge-Ogi A, Orian-Rousseau V, Brabletz S, Brabletz T, et al: A novel ZEB1/HAS2 positive feedback loop promotes EMT in breast cancer. Oncotarget 8: 11530-11543, 2017.

41. Liu G, Liu YJ, Lian WJ, Zhao ZW, Yi T and Zhou HY: Reduced BMP6 expression by DNA methylation contributes to EMT and drug resistance in breast cancer cells. Oncol Rep 32: 581-588, 2014.

42. Larue L and Bellacosa A: Epithelial-mesenchymal transition in development and cancer: Role of phosphatidylinositol 3' kinase/ AKT pathways. Oncogene 24: 7443-7454, 2005. 